\title{
SISTEMATIZACIÓN DE RESULTADOS DEL PROGRAMA DE MEJORAMIENTO CUALITATIVO DE LA EDUCACIÓN EN INSTITUTOS TÉCNICOS PROFESIONALES DE COSTA RICA
}

José Ramírez Alfaro ${ }^{I}$

\section{Resumen}

El presente artículo expone los resultados obtenidos a través del Programa de Mejoramiento Cualitativo de la Educación en Institutos Técnicos Profesionales costarricenses distribuidos en cinco regiones: Zona Norte, Limón, Zona Sur, Pacífico Central (Aguirre) y Santa Cruz.

Esta experiencia se planteó como proyecto piloto, desarrollando desde los Institutos Técnicos, un diagnóstico situacional y un plan de acciones de intervención para el mejoramiento, en especial, de infraestructuras y equipos didácticos. Posteriormente, se llevó a cabo una etapa de planificación estratégica, de revisión y desarrollo curricular y de planificación táctica o de mediano plazo. Es una propuesta metodológica para el mejoramiento cualitativo de la educación en estos Institutos Técnicos Profesionales.

Palabras claves: mejoramiento cualitativo, educación.

\section{Abstract}

This article shows results obtained in the Program of Qualitative Improvement of Education in Costa Rican Technical Institutes of five different regions: North Region, Limon, South Region, Central Pacific (Aguirre) and Santa Cruz.

This experience was designed at the Technical Institutes as a pilot project, developing situational diagnoses and interventional action plans for the improvement, specially, of infrastructure

Especialista de Educación y Capacitación y Coordinador del Centro de Capacitación a Distancia del Instituto Interamericano de Cooperación para la Agricultura (IICA). Coordinador de Capacitación y Vida Estudiantil del Centro Agronómico Tropical de Investigación y Enseñanza (CATIE).

Recibido: 3 de noviembre-2006 A Aprobado: 15 de noviembre-2006 
and didactic equipment. Subsequently, a planning stage of verification, curriculum development and tactic planning was carried out. This is a methodological proposal for the qualitative impro- vement of education in these technical professional institutes.

Keywords: qualitative improvement, education.

\section{Introducción}

$\mathrm{E}$

n Costa Rica, al igual que en toda Latinoamérica, existe la necesidad clara educación general, sobre todo en aquellos de formación técnica productiva. En un alto porcentaje, los programas de estudios de la mayoría de los centros educativos -desde la primaria hasta la universidad-carecen de relación directa con el medio en donde se encuentran y con las exigencias de conocimientos de las actuales sociedades, crecientes y cambiantes.

Las instituciones educativas, que decidan enfrentar los cambios acelerados que ocurren en la sociedad, deben tener una clara dirección de su propio futuro y del aporte que necesita la sociedad en cuanto a la formación de los ciudadanos. Es necesario que los centros de formación técnica, en especial los Institutos Técnicos Profesionales, realicen permanentemente estudios tanto del contexto donde se desarrollan, como de sus recursos y procesos internos, identificando la razón de ser, por qué existen y cuál es su función en la sociedad. Para ello, es preciso determinar cuáles son las oportunidades, amenazas, fortalezas y debilidades que poseen. Al definir con claridad su papel y su objetivo, cada institución podrá identificar al estudiante como el principal objetivo, de tal manera que al brindarle una oferta educativa atractiva y de calidad, este pueda desarrollar sus capacidades cognoscitivas, psicomotrices y psicoafectivas, aunado a un crecimiento personal integral, que le permita ser una persona que pueda transformar la sociedad en busca de la equidad, justicia social y sustentabilidad.

Por este motivo, en Costa Rica, específicamente en los Institutos Técnicos Profesionales, existe un marcado interés por elevar los niveles de calidad y pertinencia de estos institutos en general y en las distintas unidades académicas, con miras a asegurar estándares de calidad, conducentes a procesos de evaluación y acreditación de la oferta académica que demanda el desarrollo social y económico costarricense.

Con esta política, es importante aprovechar el momento y plantear propuestas dirigidas a desarrollar estrategias didácticas, cambios en las estructuras curriculares y transformaciones en las prácticas didáctico-productivas, 
que mejoren el funcionamiento de los centros educativos; encontrando así alternativas para desarrollar una alta dosis de compromiso y entrega de los participantes en el acto educativo (profesores, estudiantes, padres de familia y autoridades universitarias). Así, los esfuerzos conjuntos, el uso adecuado de recursos y el diseño de nuevas estrategias, generan resultados positivos en cuanto a rendimiento académico, producción y formación de un profesional más capacitado y más humano; un profesional que funcione como agente de cambio para sus comunidades y posea la visión de mejorar, desde su perspectiva laboral, su estilo de vida, el de su familia y el de las futuras generaciones de la sociedad a la cual pertenece.

\section{Metodología}

Este trabajo se desarrolló en Institutos Técnicos Profesionales de cinco regiones a saber: Zona Norte, Limón, Zona Sur, Pacífico Central (Aguirre) y Santa Cruz. Como se mencionó, el proyecto comprendió la cooperación del IICA, colaboración mediante la cual se ejecutó la fase de mejoramiento cualitativo, desarrollando las capacitaciones y los asesoramientos necesarios para obtener como resultado un equipo institucional, capacitado en planificación estratégica, revisión y desarrollo curricular y planificación tácticooperativa o de mediano plazo, según se representa en el siguiente modelo:

\section{Modelo metodológico de transformación académica}

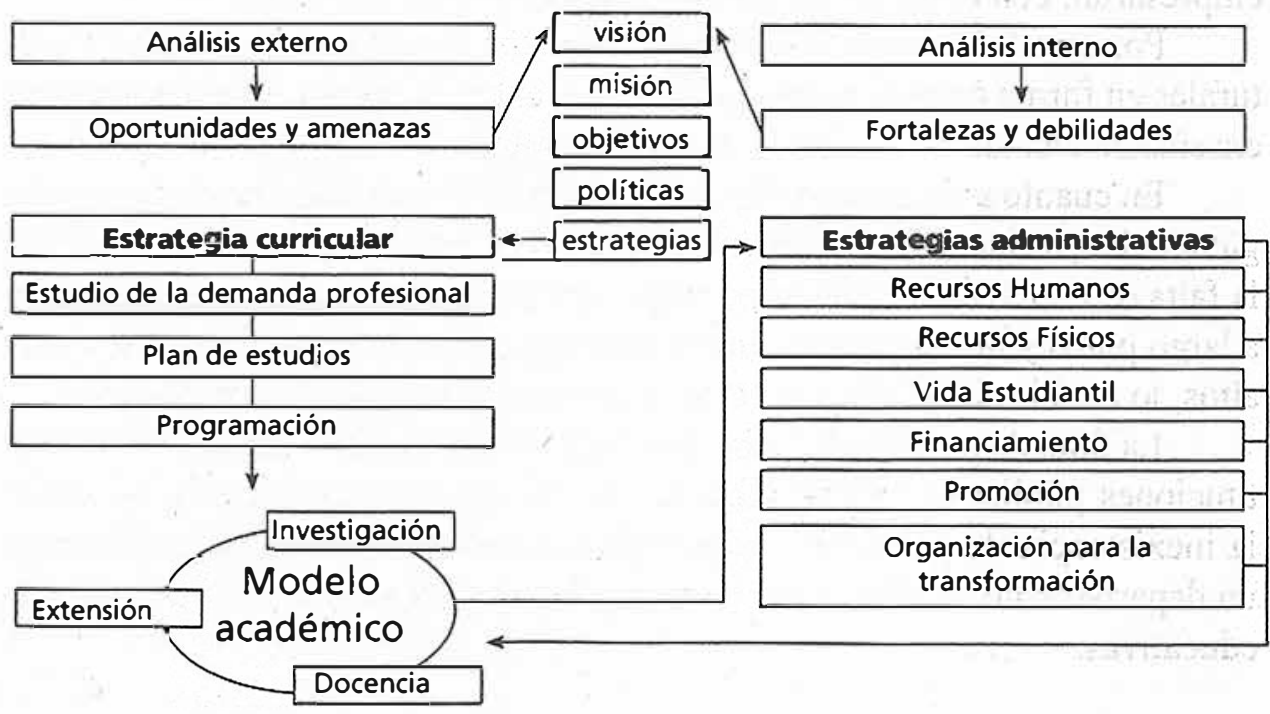




\section{Resultados de la fase de mejoramiento cualitativo}

El análisis del entorno de los Institutos Técnicos Profesionales se inició con la revisión de dichas dimensiones, con el fin de identificar las oportunidades y amenazas que, de una u otra manera, repercutirán en su desarrollo.

Mediante el trabajo grupal, se identificaron los aspectos y variables demográficas, sociales, culturales, geográficas, económicas, jurídicas y tecnológicas que mejor caracterizan el entorno. A continuación, se presenta una descripción de los factores externos considerados como críticos que favorecen el desarrollo futuro de estas instituciones.

Respecto al tema financiero, existen leyes y normativas de financiamiento de la educación tendientes al desarrollo de proyectos agropecuarios, comerciales e industriales que favorecen el desarrollo comunal, donde la principal fuente de ingresos es el presupuesto generado por la Ley 7372 , los dineros girados por DANEA con base en la Ley 7668, los fondos becarios de FONABE y el ingreso aportado por los gobiernos locales (municipalidades) a las instituciones; mientras que fuentes externas aportan ayudas económicas: Juntas Administrativas, asociaciones de padres de familia, IMAS, INA, empresas privadas de cada zona, sector comercial, entre otras.

La realización de mesas empresariales les permite a los administradores del sector empresarial expresar sus necesidades laborales. Es señalada como otra oportunidad significativa, pues cuenta con una oferta variada de especialidades, y se establecen a partir de la demanda solicitada por el sector empresarial, con el fin de garantizarle al discente un empleo.

Por otro lado, se aprovechan los recursos tecnológicos, sociales y culturales en forma óptima e interactúan como entes de apoyo al desarrollo de la enseñanza técnica y académica de cada instituto.

En cuanto a los factores limitantes para el funcionamiento de cada colegio técnico, se han de mencionar los siguientes, que dependen de otros entes: la falta de contenidos económicos para desarrollar proyectos autosostenibles a largo plazo y las fuentes de financiamiento existentes tienen intereses muy altos, lo cual limita el desarrollo de inversiones en proyectos productivos.

La falta de políticas de descentralización y desconcentración de las instituciones públicas y privadas es otra de las amenazas señaladas, así como la inexistencia de políticas, que permitan a cada Dirección Regional, tener un departamento de educación técnica y los cambios constantes de políticas educativas. 
No existe coordinación entre las empresas y las instituciones públicas, de manera que no tienen ninguna relación con las necesidades laborales de estas y de las aspiraciones de los mismos estudiantes.

El análisis de los Institutos Técnicos Profesionales consistió en hacer una evaluación interna de cada uno de ellos, para identificar sus fortalezas y debilidades, frente a las oportunidades y amenazas que han sido identificadas en el análisis del entorno. Se entiende como fortaleza aquel factor interno que favorece el aprovechamiento de las oportunidades y/o protege de amenazas; lo opuesto se definió como debilidad.

En este análisis, se contemplaron como recursos y procesos determinantes del desarrollo institucional educativo los siguientes: recursos financieros, recursos humanos y físicos, procesos organizacionales, procesos de admisión, de inducción y productos.

Una de las fuentes económicas comunes son los recursos provenientes de la Ley 7372, los fondos becarios de FONABE, los fondos de DANEA y las becas por transporte estudiantil que brinda el Ministerio de Educación Pública; todos son fondos oficiales y permanentes, cuya inversión está sujeta a la información que suministra cada institución.

En estas instituciones, se da la existencia de personal calificado, capacitado y comprometido con la educación técnica, que tiene contacto con tecnología de punta; en cuanto a materiales didácticos, sí hay bibliotecas cuya bibliografía está relacionada con las especialidades impartidas, no obstante, carecen de actualización, mas no de contenidos. Los colegios cuentan con servicios adiciones tales como Internet, servicio de fax y servicio de correo electrónico.

En cuanto a factores débiles, puede mencionarse que se da un mal proceso de planificación por parte de los entes administrativos e inadecuado manejo y distribución de los recursos, de manera que se limita el crecimiento sostenido de esas instituciones.

De igual forma, existe personal técnico interino, lo que promueve la rotación, lo que impide la continuidad en el proceso de enseñanza-aprendizaje y reduce las probabilidades de darle seguimiento a los proyectos; aspectos que se convierten en factores desmotivantes entre el estudiantado.

Otro factor debilitante es que los estudiantes no reciben formación integral, con conocimientos y manejo técnico que permita aprovechar las oportunidades y alternativas del mercado laboral, con una participación como oferentes o demandantes. 


\section{Visión de desarrollo de la educación técnica}

La visión describe el escenario que, a largo plazo, se plantea en la institución, bajo el supuesto de haber superado las debilidades y aprovechado las fortalezas, sacando ventaja de las grandes oportunidades del entorno y estableciendo una sólida protección ante las amenazas del contexto institucional.

Seguidamente se mencionan los elementos comunes generales que se presentan:

Ser una institución del más alto grado de competitividad a nivel nacional, que se visualiza como un ente formador de manera holística, para ofrecer a los educandos -sin distingo de género- una educación técnica con capacidad para desarrollar una educación técnica en forma integral, científica y tecnológica de calidad y excelencia como una herramienta para lograr el desarrollo sostenible, involucrando la conservación del medio ambiente y las necesidades de cada región, para una mejor competencia dentro del proceso de globalización.

\section{La misión de desarrollo de la educación técnica}

Se plantean, a continuación, aquellos elementos a los que las instituciones han identificado como componentes de su misión:

Ciclo Diversificado y Técnico Medio, capaces de formar profesionales a nivel de técnicos medios que cuenten con valores espirituales, éticos, morales y sociales y con la actitud necesaria para satisfacer la demanda de personal técnico requerido por el entorno, al contribuir con sus conocimientos en el desarrollo de las empresas establecidas o en la creación de las mismas para el progreso social y económico.

\section{Objetivos de la educación técnica}

En términos generales y desprendidos de los grandes propósitos de la misión, se propusieron los siguientes objetivos:

- Buscar nuevas formas de inserción laboral para la ubicación de los estudiantes. 
- Fomentar la cooperación entre los colegios para la solicitud y aprovechamiento de capacitaciones coordinadamente con el MEP.

- Establecer un vínculo de cooperación entre los colegios y los empresarios.

- Investigar constantemente los cambios en el perfil de los estudiantes, que demandan los empresarios.

\section{Politicas de la educación técnica}

Consecuentemente con el planteamiento estratégico y después de considerar las orientaciones conducentes al logro de los objetivos institucionales, se propusieron las siguientes políticas que guiarían los procesos de formación:

- Establecer parámetros que permitan identificar el docente idóneo acorde con el desarrollo de la visión y la misión.

- Mejoramiento continuo de la enseñanza, mediante capacitación permanente al personal docente y administrativo.

- Fortalecimiento de valores y actitudes en los docentes.

- Atención a la vida y asuntos estudiantiles.

- Equidad de género en todo el contexto institucional.

- $\quad$ Promoción constante de futuros egresados.

- Altemativas y valores educativos que propicien el desarrollo socioeconómico en los jóvenes.

- Proyectos comunitarios relacionados con la formación que están recibiendo los estudiantes.

- Alianzas estratégicas interinstitucionales para maximizar recursos y conocimientos existentes.

- Contribuir a modemizar la oferta educativa de los colegios técnicos, acorde con las necesidades y posibilidades de la institución y de acuerdo con su entomo.

- Establecer lineamientos en materia de propuesta educativa.

- Establecer proyectos didácticos productivos que permitan el fortalecimiento de las especialidades, mediante la aplicación de la teoría-práctica. 


\section{Lineamientos estratégicos para el desarrollo de la educación técnica}

Después de plantear los objetivos estratégicos y de proponer las políticas que servirán de marco formativo para el plan de desarrollo en los Institutos Técnicos Profesionales, se procede a:

- Conformación de convenios o alianzas estratégicas con instituciones estatales como el INA, IMAS, Instituto Tecnológico de Costa Rica, entre otras, que fortalezcan el proceso de enseñanza-aprendizaje.

- Uso de la informática como herramienta: adquisición de equipo tecnológico de punta que colabore con la labor docente y con el proceso de enseñanza-aprendizaje.

- Presentación de proyectos de adquisición de equipo a la comisión de la Ley 7372.

- Información al Departamento de Educación Técnica respecto a estudios de inserción laboral, que permitan seleccionar, en una forma objetiva, la oferta académica de la institución.

\section{Evaluación y desarrollo de una propuesta de mejoramiento curricular}

\section{Perfil de desempeño académico del técnico}

Luego de realizar una serie de consultas dirigidas a informantes claves, estos dieron su opinión en cuanto a las áreas específicas que deben ser reforzadas en los programas de estudio:

- Confianza y seguridad en sí mismo, para asumir responsabilidades y toma de decisiones.

- $\quad$ Organización, habilidad negociadora y capacidad para las relaciones sociales y para el trabajo en equipo.

- Flexibilidad mental y predisposición para asumir los cambios que exige el mercado de trabajo.

- Capacidad de comunicación, demuestra calidad en la expresión oral y escrita, así como en el manejo del idioma inglés.

- Colaboradores en la prestación de bienes y servicios a la comunidad.

- Forma un Técnico Medio que sea emprendedor e innovador.

- Utiliza los medios científicos y tecnológicos aplicados a las tareas propias de la especialidad, para contribuir a la competitividad, calidad y desarrollo del país. 


\section{Plan de estudio para la formación técnica profesional}

El plan de estudios es el ordenamiento de las asignaturas y cursos, que debe llevar el estudiante desde el momento de ingreso hasta que se gradúa. Para definir los cursos y asignar el número de créditos, se parte de las derivaciones que se desprenden de las capacidades, la definición de objetivos, contenidos y el tiempo que requiere cada objetivo para ser alcanzado por el o la estudiante.

El currículum actual tiene muchos contenidos teóricos que prácticamente son desarrollados por las materias de carreras de agronomía; sin embargo, son poco prácticos. Por ello, es fundamental que en la organización del currículo, se enfatice la parte práctica, de manera que sea alrededor de un $60 \%$ práctico y un $40 \%$ teórico. Por esta razón, se deben realizar cambios sustanciales, principalmente, en las materias básicas para que haya concordancia con el nivel académico y las funciones que el profesional vaya a desempeñar fuera del Instituto Técnico Profesional.

En síntesis y como resultado del análisis de las competencias y roles que debe asumir el profesional, es evidente que el mercado laboral demanda un técnico con mayores habilidades prácticas, que le permitan desarrollar proyectos productivos, antes que sólo poseer muchos conocimientos teóricos. Resulta, entonces, recomendable elaborar una nueva propuesta del programa de estudio que en general presente características como las siguientes:

- $\quad$ El proceso de aprendizaje debe ser orientado para la vida y en la validación de los conocimientos adquiridos en situaciones específicas de la vida real.

- Los fundamentos de sostenibilidad deben ponerse en práctica en todas las áreas de producción.

- Las unidades de estudio deben orientarse a la producción de bienes y servicios, principalmente en los niveles de undécimo y duodécimo años.

- El currículo de la especialidad debe brindar las suficientes bases teórico-prácticas para que el egresado pueda desarrollar iniciativas de producción en nuevas áreas.

- Se debe estimular la visión y mentalidad empresarial (autogestionaria), donde la innovación y la creatividad sean la constante en el proceso de aprendizaje.

- Una propuesta de renovación del Plan de Estudios en la especialidad. 


\section{Estrategias para el desarrollo organizacional}

Después de plantear los objetivos estratégicos y de proponer las políticas que servirán de marco formativo para el plan de desarrollo en los Institutos Técnicos Profesionales, se procede con la elaboración de las estrategias o definición de los pasos para alcanzar objetivos y cumplir las políticas.

De manera general, las estrategias mencionadas por los Institutos Técnicos Profesionales, en el área de desarrollo de los Recursos Humanos, se sintetizan en el financiamiento de la capacitación continua de los docentes en rescate de valores y equidad de género.

Para el mejoramiento de los recursos físicos, se plantearon diversas estrategias en las cuales destacan, en especial, las obras de infraestructura y la orientación de los recursos para equipar y consolidar la infraestructura para cada especialidad, de acuerdo con los mínimos requerimientos. También en la planificación de la producción de las fincas, según los requerimientos de desarrollo.

Se debe atender las necesidades estudiantiles, mediante la sensibilización de la equidad de género en la inserción de los y las estudiantes en diversas especialidades y servicios estudiantiles de calidad. Para lograr una adecuada divulgación comunal sobre el acontecer institucional de los Institutos Técnicos Profesionales, se promoverá la inserción institucional en programas sociales.

Entre los principales proyectos que plantearon los Institutos Técnicos Profesionales para su financiamiento, resaltan la identificación y entrega de los recursos económicos necesarios para su buen desempeño, y la captación de recursos mediante proyectos de inversión para la producción.

\section{Estrategia de organización para el cambio}

La implementación del proyecto implica cambios en la estructura organizativa de cada institución. Estos cambios permitirán y facilitarán el desarrollo y ejecución de los diversos proyectos planteados por cada una de las áreas que componen el planeamiento:

- Eficiencia administrativa.

- Integración de una comisión con miembros del comité de bienestar estudiantil, padres de familia, orientación y profesores guías para realizar estudios socioeconómicos. 
- Ayuda técnica y legal especializada en diferentes entes públicos y privados que contribuyan con la administración de los Colegios Técnicos.

- Calidad en el servicio al cliente.

- Enlace comunal.

- Definir un solo reglamento.

- Mejoramiento continuo.

- Buenas relaciones humanas.

\section{Prospectiva de inversión requerida para implementar programas y pro- yectos de desarrollo en la educación técnica}

En este aspecto, es importante mencionar que el presupuesto elaborado por cada Instituto Técnico Profesional, puede variar en el momento de la ejecución de los proyectos; sin embargo, representa una excelente guía en el análisis de la inversión requerida para alcanzar el desarrollo exitoso de los programas planteados.

Ciertos Institutos no plantearon presupuestos y/o consideraron que algunos proyectos no requieren de inversiones importantes; pero necesitan compromiso e identificación con la institución por parte de los docentes, administrativos, colaboradores y de los mismos estudiantes.

Es importante mencionar que algunos de los costos por rubros de proyectos fueron incrementados, por lo que en una segunda etapa, se requiere revisar esos presupuestos con criterio económico financiero. Esta es una recomendación muy importante, sobre todo porque existen proyectos cuya inversión es bastante alta, donde definitivamente el planteamiento para cinco años no es suficiente.

\section{Conclusiones}

El objetivo inicial se ha alcanzado considerablemente bien; al existir un ambiente de trabajo diferente y desde que se inició el proyecto, se han llevado a cabo cambios significativos en los procesos de formación y convivencia en los Institutos Técnicos Profesionales que participaron. Además, se consolidó el respaldo por parte del Ministro de Educación y su despacho, así como de la Dirección de Educación Técnica.

Desde el punto de vista del mejoramiento de la calidad y la pertinencia institucional, se puede afirmar que el objetivo se cumplió, mediante la conclusión de las capacitaciones ofrecidas por el IICA y la preparación final 
de una propuesta de desarrollo institucional por cada Instituto Técnico Profesional, la cual comprende planes de desarrollo curricular, estrategias de desarrollo institucional y planes tácticos de mediano plazo.

En cuanto a las necesidades de estrategias administrativas para canalizar las fuentes de financiamiento en busca del mejoramiento de la infraestructura de los Institutos Técnicos Profesionales, se alcanzó un $90 \%$ de cumplimiento de las necesidades detectadas.

A través de las consultas a los diferentes grupos de informantes claves, en especial de los empresarios, se logró dar el primer paso en el desarrollo de vínculos de comunicación y retroalimentación que ya son reales, pero que deben fortalecerse; por lo tanto, el objetivo se cumplió tal y como se planteó desde un inicio.

Se desarrolló una propuesta de Plan Estratégico y de Desarrollo Institucional, que comprende un análisis del contexto, un análisis interno, la visión, misión, objetivos, políticas y lineamientos estratégicos, que determinarán el rumbo de las instituciones participantes.

En términos generales, la experiencia llevada a cabo en estos cinco Institutos Técnicos Profesionales se puede definir y valorar como exitosa, ya que se conformaron equipos de trabajo en las instituciones, que ahora capacitados pueden garantizar un trabajo de mayor calidad y eficiencia en cada institución.

Además, se logró iniciar un cambio en la actitud de los docentes y otros colaboradores de los Institutos Técnicos Profesionales e igualmente importante es la inquietud que se deja acerca del cambio constante y el mejoramiento continuo de los procesos de enseñanza-aprendizaje.

\section{Recomendaciones}

Las siguientes recomendaciones están planteadas desde dos puntos de vista:

a. Requerimientos o condicionamientos necesarios para que las propuestas planteadas puedan ser desarrolladas exitosamente.

b. Recomendaciones de planificación y organización para mejorar la realización de la metodología experimentada en los Institutos Técnicos Profesionales. 
Con base en lo anterior, se necesita, de manera general, orientar y facilitar procesos de seguimiento y evaluación de la calidad institucional para el cumplimiento de criterios, estándares e indicadores de calidad para la acreditación educativa.

Es fundamental establecer un sistema de consecución de recursos económicos para financiar los proyectos institucionales no financiados por la Ley 7372. Algunas de las estrategias planteadas en los proyectos pueden funcionar, y lo harán mejor si desde entidades ministeriales se tiene un mayor apoyo.

Lograr mayor flexibilidad por parte del Ministerio de Educación acerca de la organización, evaluación y manejo de los recursos en las instituciones educativas, en especial en situaciones como el nombramiento y selección de docentes y, principalmente, de directores de estas instituciones. Esto con el fin de erradicar la práctica de nombramientos políticos en los puestos vacantes del Ministerio de Educación Pública.

En este mismo sentido, es imprescindible que en cuestión de nombramientos, se busque en todo momento la continuidad del personal docente existente en la institución y que antes de enviar profesionales en propiedad, se realice un análisis previo del personal docente que se sustituirá y se valore si debe o no ser sustituido. Lo anterior tomando en cuenta aspectos como: estudios superiores por obtener, profesionalismo, especialidad, procedencia, entre otros.

Establecer mecanismos que permitan que las pruebas universitarias sean menos ambiguas, de manera que no exista un divorcio entre el sistema de evaluación del aula y la evaluación de las pruebas universitarias.

Se requiere un mayor compromiso de todos los integrantes de la institución para un mejor logro de los objetivos, así como la integración de la comunidad, padres de familia y los estudiantes. Asimismo, es necesario hallar los mecanismos adecuados para la integración del equipo de trabajo y crear una estructura orgánica innovadora que responda al cambio.

Es preciso contar con los desembolsos económicos de la Ley 7372 de forma oportuna y efectiva, según el cronograma propuesto para cada proyecto, con el fin de aspirar a mejores oportunidades de desarrollo.

La permanencia y el fortalecimiento de los proyectos de Educación Técnica siempre se han considerado como vehículos de desarrollo humano sociales y tecnológicos. El establecimiento y fortalecimiento de esta propuesta brinda las pautas para crear modelos autóctonos que brindan soluciones a 
las necesidades propias del entorno y hasta pueden representar un verdadero modelo de desarrollo en materia de formación y educación técnico-académica, pues existe identificación y compromiso concreto con los sectores involucrados.

Desde el punto de vista de las recomendaciones metodológicas, se puede mencionar, en primera instancia, la importancia de seleccionar el tutor regional que establece la Dirección Regional de Educación Técnica; esto porque no puede ser un recargo de trabajo, en realidad comprende un trabajo casi de tiempo completo.

Igualmente importante es la selección del tutor institucional del Instituto Técnico Profesional y en la medida de lo posible, sería conveniente capacitar a más de un tutor por Instituto. Las comisiones institucionales deberán conformarse por personas comprometidas e identificadas con el mejoramiento del proceso de enseñanza.

Es fundamental que los tutores seleccionados no falten a las capacitaciones y, mucho menos, a las visitas de seguimiento a los Institutos Técnicos Profesionales. La representación de las autoridades es muy importante, ya que es una manera de formalizar la participación en el proyecto.

Finalmente, otra de las recomendaciones es que el proceso inicie con una inducción por parte de la Dirección Regional, a toda la comunidad cercana al Instituto Técnico Profesional que estará vinculada a los proyectos. Esto permitiría una mayor participación, se disminuirian las reacciones defensivas o de resistencia al cambio por parte de los miembros de la comunidad, de docentes, administrativos, colaboradores y hasta los mismos estudiantes; en este sentido, la información directa y clara es fundamental. 


\section{REFERENCIAS}

Ackof, R.; Vergara, E.; Jamshid, G. (1986). Guia para controlar el futuro de la empresa. México: LIMUSA.

Aldana, E. (s.f.). Guia instruccional. Universidad de Los Andes, SAGE.

Blanchard, K. (1997). Administración por valores. Bogotá, Colombia: Norma.

Borges, J.; Escobar, M.; Palomino, J.; Saldaña, R.; De Souza, J. (1995). Planificación estratégica en la administración de la investigación agropecuaria. Fascículo 2. Netherlands: ISNAR.

Bowman, C. (s.f.). La esencia de la administración estratégica. s. n. t.

Castelán, B. (1985). Planeación estratégica y control de gestión. México: ECASA. Cedeño, A. (1993). Administración de la empresa. San José, Costa Rica: EUNED.

Drucker, P. (1995). Gerencia para el futuro. Bogotá, Colombia: Norma.

Grouard, B. y Meston, F. (1996). Reingenieria del cambio. México: ALFAOMEGA. Hamel, G. y Prahalad, C. (1995). Compitiendo por el futuro. Barcelona, España: Ariel.

Harrington, H. (1993). Mejoramiento de los procesos de la empresa. Bogotá, Colombia: Mc Graw Hill.

Hernández, S. (s.f.). Los retos de la dirección universitaria en la década de los 90 .

La Habana: Instituto Superior Politécnico José A. Echeverría.

Morgan, G. (1995). Images of organization. California: SAGE.

Morrisey, G. (1996). Planeación estratégica. México: Prentice Hall.

Morrisey, G. (1996). Planeación táctica. México: Prentice Hall.

Muñoz, C. (1995). Análisis y propuestas para la planeación educativa. Washington: OEA.

Ogliastri, E. (1985). Manual de planificación estratégica. Bogotá, Colombia: Tercer Mundo.

Ohmae, K. (1989). La mente del estratega. México: Mc Graw Hill.

Pfeiffer, W.; Goodstein, L.; Nolan, T. (1989). Shaping strategic planning. Frogs, dragons, bees and turkey tails. Illinois, U.: Scott Foreman.

Ramírez, E. (1995). Hablemos de gerencia: un enfoque futurista. San José, Costa Rica: Alma Máter.

Sallenave, J. (1985). Gerencia y planeación estratégica. Bogotá, Colombia: Norma.

Senge, P. (1992). La quinta disciplina. Barcelona, España: GRAMICA.

Senge, P. (1995). La quinta disciplina en la práctica. Barcelona, España: GRAMICA.

Senge, P. (s.f.). La danza del cambio. Barcelona, España: GRAMICA. 
Steiner, G. (1968). The critical role of the management in long-range planning. Arizona: Review.

Steiner, G. (1978). Planeación estratégica, lo que todo director debe saber. México: Continental.

Tamasko, R. (1996). Repensar la empresa. Barcelona, España: Paidós.

Wentiling, T. (1992). Plannning for efective trainnnig. Guide to curriculum development. Washington, D.C.: FAO. 